\title{
Evaluación productiva de 16 variedades de sorgo forrajero en la zona sur de Honduras
}

\author{
Edgar Osiris Carranza Espinal* ${ }^{*}$ Alberto Morán
}

\section{RESUMEN}

La producción de granos básicos es uno de los rubros de mayor importancia social y económica en el país, representa el 13.3 del PIB agrícola y genera 300.000 empleos permanentes. Los agricultores nacionales de sorgo reportan un bajo desempeño del cultivo debido a la carencia de nuevos materiales genéticos que se adapten a las condiciones agroecológicas en las áreas de siembra, los materiales disponibles son de doble propósito: de origen criollo y de pobre desempeño productivo; por lo que son necesario nuevos materiales genéticos accesibles a los pequeños productores y con altas características de desempeño productivo, para contribuir a la reducción de la amenaza de inseguridad alimentaria y nutricional en las comunidades rurales del país. El objetivo del estudio fue comparar el desempeño productivo de 16 materiales de sorgo forrajero durante la época de postrera en la Estación Experimental, La Lujosa, de los años 2010 y 2011, para seleccionar nuevas variedades de sorgo. Se usaron cuatro parcelas experimentales con 16 tratamientos y cuatro repeticiones, se empleó el modelo estadístico de Diseño de Bloques al Azar, se analizó la producción de forraje, contenido nutricional del forraje, día a flor, altura de planta y producción de grano. La variedad que mostro mejor desempeño productivo en forraje fue Centa S-3, seguido de las variedades C10925 y C10947. La variedad que produjo mayor cantidad de grano fue Centa S-3, seguido de las variedades C10947 y C1938.

Palabras clave: Variedad, seguridad alimentaria y nutricional, forraje, pequeños agricultores, características de desempeño.

\section{ABSTRACT}

The basic grain production is one of the areas of greatest social and economic importance in the country, accounting for 13.3 of the agricultural GDP and generates 300,000 permanent jobs. National Farmers reported sorghum crop underperforming due to lack of new genetic material to suit the agroecological conditions in the areas

* Universidad Nacional Autónoma de Honduras. Centro Universitario Regional del Litoral Pacífico. Departamento de Acuicultura y Biología Marina. edgar.carranza@unah.edu 
of planting materials available are dual purpose of Creole origin and poor productivity performance, so the need to have new genetic material accessible to small producers and high production performance characteristics, to help reduce the threat of food and nutrition insecurity in the country's rural communities. The objective of the study was to compare the productive performance of 16 forage sorghum materials postrera during the Experimental Station The Lavish the years 2010 and 2011, to select new varieties of sorghum. Four experimental plots were used with 16 treatments and four replications was used statistical model randomized block design was analyzed forage production, forage nutritional content, days to flower, plant height and grain yield. The strain that showed better performance in forage production was Centa S-3, followed by CI0925 and Cl0947 varieties. The strain that produced the most grain was Centa S-3, followed by Cl0947 varieties and Cl938.

Key words: Variety, nutrition and food security, fodder, small farmers, performance characteristics. 


\section{INTRODUCCIÓN}

La producción de granos básicos es uno de los rubros de mayor importancia social y económica en el país, representa el 13.3 del PIB agrícola y genera 300.000 empleos permanentes (SAG, 2010). Los granos básicos conforman el 35\% de la dieta diaria del hondureño. En la zona rural las familias con mayores índices de pobreza, el maíz, frijol y el sorgo, conforman más del $60 \%$ de su dieta alimenticia y animal (IICA, 2010). Los agricultores nacionales de sorgo reportan un bajo desempeño del cultivo, debido a la carencia de nuevos materiales genéticos que se adapten a las condiciones agroecológicas de las áreas de siembra. Los pequeños agricultores tradicionalmente siembran sorgo o maicillo que sirve de doble propósito (grano y forraje), para la alimentación de animales con el forraje del cultivo y la producción del grano.

Los materiales disponibles de sorgo de doble propósito para los pequeños agricultores, generalmente son de origen criollo y de pobre desempeño productivo. Existen híbridos y variedades de sorgo con mejores características que el del origen criollo, pero estos materiales son comercializados por compañías transnacionales a un precio que no es accesible al pequeño productor (Sandoval, 2011). El sorgo es una planta de días cortos, este cereal es capaz de adaptarse a climas secos, calientes, húmedos e incluso en condiciones de salinidad y en suelos de baja fertilidad. Como forraje su valor energético es alto. El ensilaje de sorgo es utilizado para alimentar el ganado en las épocas de baja producción de las pasturas (Díaz, 2011).

A pesar que el sorgo es un cultivo adaptado a climas secos, que se siembra para el consumo humano, sirve de alimento para animales y tiene alto potencial para la producción de biocombustible, se carece de nuevos materiales genéticos accesibles a los pequeños productores y con altas características de desempeño productivo para contribuir a reducir la amenaza de la inseguridad alimentaria y nutricional en las comunidades rurales del país. Actualmente los productores nacionales de sorgo registran rendimientos promedios de 1,620 kg por hectárea de grano (Rodríguez, 2012), y la tendencia es la reducción de este margen productivo debido a factores ambientales, recursos agrícolas limitados y bajo desempeño productivo del material genético usado por el productor.

En el país existen alrededor de 15 mil productores de sorgo que han sido beneficiados con el bono tecnológico productivo (SAG, 2010), que consiste en la dotación de semilla mejorada y fertilizante a los pequeños productores. Pero la 
semilla de sorgo que se usa en el bono tecnológico es para la producción de grano y existe en el país desde el año 1983 (IICA, 2010). Así mismo, la variabilidad climática registrada en los últimos cinco años, influye negativamente en las producciones del cultivo de sorgo y maicillo criollo, y con la disponibilidad de nuevos materiales genéticos se espera que estos soporten los efectos de la variabilidad climática y mantengan sus características productivas. La calidad del forraje de sorgo se puede aumentar mejorando las características de desempeño del cultivo como sabor de grano, la disponibilidad de nutrientes disponibles en la materia seca de la planta, producción de grano y forraje, precocidad, altura de planta y tamaño de la panoja (Urquiza, 2001).

La digestibilidad de la materia seca, el contenido energético y el valor de NDT de los sorgos son siempre inferiores a los del maíz (Browning \& Lusk, 1966). Por esta razón a través de los años los genetistas han tratado de cambiar o igualar estas diferencias. El total de nutrientes digestibles (TND) es una medida aproximada de la digestibilidad de un alimento. Valores mayores de TND indica un mayor valor nutritivo del forraje (Valencia, 2011), a pesar que los valores de TND se basan en un análisis proximal, estos valores son muy utilizados en la alimentación animal. Existen valores que dan referencia al contenido nutricional del sorgo forrajero para la alimentación de rumiantes (Tabla 1).

\section{Tabla No. 1. Contenido nutricional del sorgo forrajero, según el Comité Internacional de Nutrición Animal.}

\begin{tabular}{|l|c|}
\hline \multicolumn{1}{|c|}{ Referencia } & Valores \\
\hline Materia seca (\%) & 23 \\
\hline TND (\%) & 63 \\
\hline NEL (Mcal//kg) & 1.42 \\
\hline Extracto etéreo & 1.6 \\
\hline Fibra cruda (\%) & 30.0 \\
\hline Calcio (\%) & 0.43 \\
\hline Fósforo (\%) & 0.36 \\
\hline
\end{tabular}

Para liberar nuevas variedades de sorgo forrajero, estas deben evaluarse en parcelas experimentales y parcelas de validación. Constantemente la Secretaría de Agricultura y Ganadería a través de la Dirección de Ciencia y Tecnología Agropecuaria (DICTA), evalúa nuevos materiales genéticos de cultivos con altas características productivas, tales como el arroz, maíz, frijol, sorgo, ajonjolí y cacahuate en estaciones y campos experimentales de DICTA (SAG, 2010). 


\section{OBJETIVOS}

\section{Objetivo General}

Comparar el desempeño productivo de 16 materiales de sorgo forrajero durante la época de postrera en la Estación Experimental La Lujosa de los año 2010 y 2011, para seleccionar nuevos híbridos y cultivares de sorgo accesibles a los pequeños agricultores del país, y contribuir a reducir la amenaza de la seguridad alimentaria y nutricional.

\section{Objetivos Específicos}

1. Encontrar las variedades de sorgo forrajero que tienen mayor rendimiento de forraje y grano probados en la Estación Experimental La Lujosa;

2. Evaluar las variedades de sorgo forrajero que tienen mayor precocidad y altura de planta;

\section{MÉTODOS}

El estudio se realizó en la Estación Experimental La Lujosa en los años 2010 y 2011 , se probaron 16 variedades de sorgo forrajero en parcelas experimentales. Para comparar producción de forraje se estableció una parcela entre los meses de junio a octubre, y para evaluar la producción de grano se estableció otra parcela entre los meses de agosto a diciembre.

\section{Diseño}

Se usó el diseño de Bloque Completamente al Azar (BCA) en las cuatros parcelas experimentales (una parcela comparó la producción de forraje y la otra la producción de grano, en cada año).Cada parcela experimental se estableció en un área total de $1,200 \mathrm{~m}^{2}$, se comparó el desempeño productivo de 16 tratamientos (variedades de sorgo forrajero) con cuatro repeticiones para cada tratamiento. Cada unidad experimental se estableció en una parcela de $14 \mathrm{~m}^{2}$, que tenían cuatro surcos de $5 \mathrm{~m}$ de largo y una separación entre surcos de $0.7 \mathrm{~m}$.

Para reducir el error del experimento por el efecto de borde, en la toma de datos y la cosecha, se consideró los dos surcos internos y $4 \mathrm{~m}$ de largo, siendo el área útil de la parcela de $5.6 \mathrm{~m}^{2}$, que en total de todo el ensayo fue de $358.4 \mathrm{~m}^{2}$. 


\section{Población}

Se analizaron 256 unidades experimentales, 64 por cada parcela experimental (16 tratamientos $\mathrm{X} 4$ repeticiones), en los dos años de estudio.

\section{Entorno}

El estudio se realizó en la Estación Experimental La Lujosa, centro de investigación de DICTA, ubicada a $12 \mathrm{~km}$ de la ciudad de Choluteca, en los $13^{\circ} 19^{\prime} 13.9^{\prime \prime}$ latitud Norte y $87^{\circ} 17^{\prime} 01.2^{\prime \prime}$ longitud Oeste, a 29 metros sobre el nivel del mar.

\section{Intervenciones}

Los ensayos tuvieron las mismas condiciones de manejo, se preparó el área de siembra con maquinaria agrícola (arado y rastra), en la siembra se usó $130 \mathrm{~kg}$ de fertilizante 18-46-0 (fosfato diamónico) por hectárea y $13 \mathrm{~kg}$ insecticida terbufos $5 \%$ por hectárea, para proteger a la semilla. A los 25 y 45 días se aplicó $130 \mathrm{~kg}$ de urea (0-46-0) por hectárea. Para el control de malezas se realizó limpieza manual a los 10 y 25 días del cultivo, y a los 40 días se aplicó el herbicida glufosinato de amonio (15\%).

Las parcelas experimentales sembradas para comparar la producción de forraje se cosecharon entre los 85 y 90 días del cultivo. En las parcelas para determinar la producción de grano se cosecharon entre los 110 y 120 días. No se usó riego, ya que se sembraron en la épocalluviosa de esos años.

\section{Análisis Estadístico}

En las parcelas experimentales para de producción de forraje, se evaluaron producción de materia fresca, producción de materia seca, producción de materia seca digestible, fibra detergente ácida y energía neta de lactancia, altura de planta y días a floración. La materia fresca, materia seca, materia seca digestible se midió en kg por hectáreas. El valor de fibra detergente ácida está dado en porcentaje, la energía neta de lactancia en mil calorías por kilogramo de materia seca, la altura de planta en metros, y los días a floración en días.

Las parcelas sembradas para determinar la producción de grano se midió el rendimiento de grano de sorgo en kilogramos por hectárea. Para el análisis estadístico se usó el BCA y para la prueba de medias se usó el modelo de Diferencia 
Mínima Significativa a un nivel de significancia del 0.05 , se usó el software estadístico Infostat $\circledast 2011$.

\section{RESULTADOS}

Se encontró diferencias estadísticas significas entre todas las variables evaluadas de los sorgos sembrados para comparar productividad de forraje y grano.

Materia fresca: se encontró diferencias estadísticas entre los materiales de sorgo $(p<0.05)$ evaluados en los años 2010 y 2011. La variedad más productiva fue Centa S-E (Figura 1) en los años de estudio, se encontró diferencias estadísticas entre cada variedad según la prueba de medias.

Figura 1. Rendimiento de materia fresca en $\mathrm{Kg}$ por hectárea de 16 variedades de sorgo forrajero, evaluadas en los años 2010 y 2011 en la Estación Experimental La Lujosa. Letras distintas indican diferencias significativas $(p<=0.05)$.

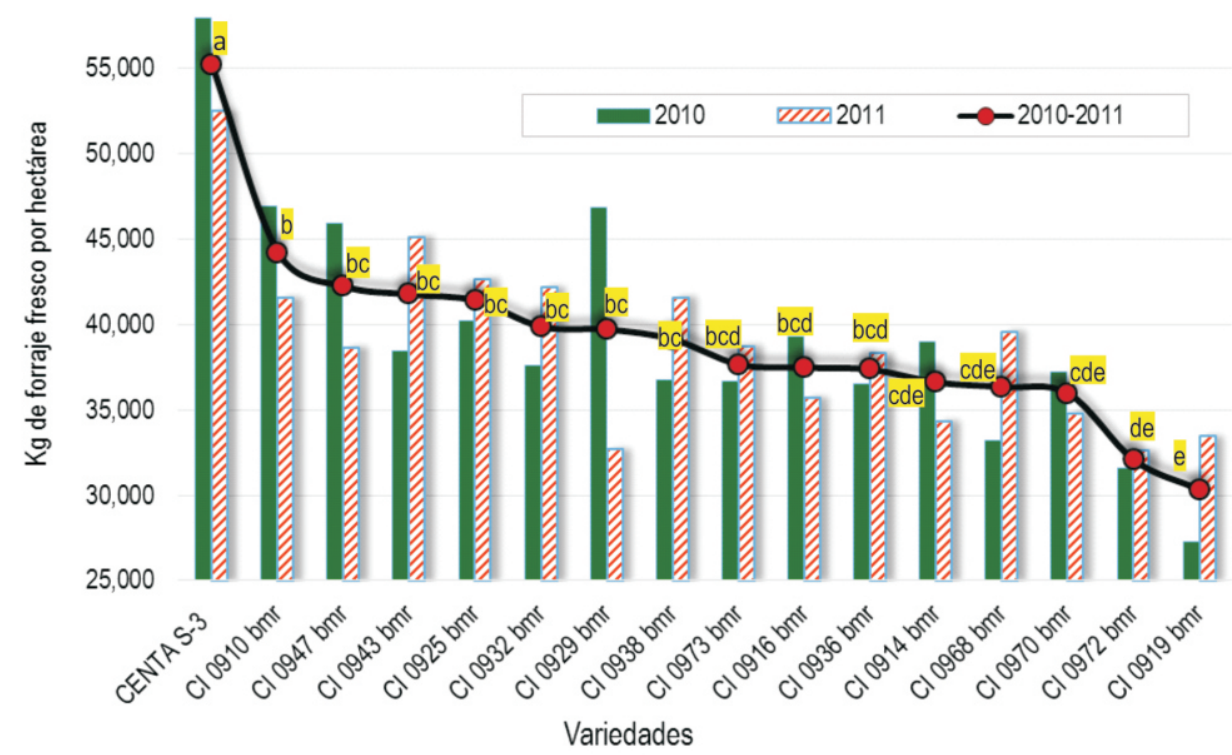

Características productivas de forraje: las variedades de los sorgos presentaron diferencias significativas de materia digestible (MSD), materia seca (MS), nutrientes digestibles totales (NDT), fibra detergente ácida (FDA) y energía neta de lactancia (ENL). 
En la tabla 2 se presentan los valores de MSD, MS, NDT, FDA, ENL de las variedades de sorgo evaluadas durante dos años consecutivos en la época de invierno en la Estación Experimental La Lujosa. La variedad que obtuvo el mayor contenido por hectárea de kilogramos de materia seca digestible es la Centa S-3 con $11,642 \mathrm{Kg} / \mathrm{ha}$, las variedades $925,947,932,936,943,973,916,914$, y 929 , tienen estadísticamente el mismo contenido en kilogramos por hectárea de materia seca digestible $(P<0.001)$.

\section{Tabla 2. Características productivas y nutritivas de 16 variedades de sorgo forrajero, sembradas en los meses de junio a agosto de los años 2010 y 2011.}

\begin{tabular}{|c|c|c|c|c|c|}
\hline \multirow{2}{*}{ Variedad } & Kg/Ha & Kg/Ha & $\%$ & $\%$ & Mcal/Kg MS \\
\cline { 2 - 7 } & MSD & MS & NDT & FDA & ENL \\
\hline CENTA S-3 & $11.642 \mathrm{a}$ & $17.457 \mathrm{a}$ & 65.9 & 28.8 & 1.52 \\
\hline $\mathrm{Cl} 0925 \mathrm{bmr}$ & $9.003 \mathrm{~b}$ & $13.291 \mathrm{bcd}$ & 68.8 & 25.2 & 1.62 \\
\hline $\mathrm{Cl} 0947 \mathrm{bmr}$ & $8.072 \mathrm{bc}$ & $13.600 \mathrm{bc}$ & 64.8 & 30.2 & 1.48 \\
\hline $\mathrm{Cl} 0932 \mathrm{bmr}$ & $7.996 \mathrm{bc}$ & $12.130 \mathrm{cde}$ & 65.7 & 29.0 & 1.51 \\
\hline $\mathrm{Cl} 0936 \mathrm{bmr}$ & $7.920 \mathrm{bc}$ & $11.863 \mathrm{cde}$ & 66.6 & 27.9 & 1.54 \\
\hline $\mathrm{Cl} 0943 \mathrm{bmr}$ & $7.866 \mathrm{bc}$ & $12.143 \mathrm{cde}$ & 62.0 & 33.7 & 1.38 \\
\hline $\mathrm{Cl} 0973 \mathrm{bmr}$ & $7.821 \mathrm{bc}$ & $11.668 \mathrm{cde}$ & 66.7 & 27.8 & 1.54 \\
\hline $\mathrm{Cl} 0916 \mathrm{bmr}$ & $7.572 \mathrm{bcd}$ & $11.368 \mathrm{cde}$ & 65.3 & 29.6 & 1.50 \\
\hline $\mathrm{Cl} 0914 \mathrm{bmr}$ & $7.441 \mathrm{bcde}$ & $11.063 \mathrm{de}$ & 67.5 & 26.8 & 1.57 \\
\hline $\mathrm{Cl} 0929 \mathrm{bmr}$ & $7.324 \mathrm{bcde}$ & $11.208 \mathrm{de}$ & 64.2 & 30.9 & 1.46 \\
\hline $\mathrm{Cl} 0970 \mathrm{bmr}$ & $7.196 \mathrm{cde}$ & $10.768 \mathrm{ef}$ & 66.5 & 31.0 & 1.54 \\
\hline $\mathrm{Cl} 0938 \mathrm{bmr}$ & $7.116 \mathrm{cde}$ & $11.994 \mathrm{cde}$ & 65.6 & 29.1 & 1.51 \\
\hline $\mathrm{Cl} 0968 \mathrm{bmr}$ & $6.581 \mathrm{cde}$ & $11.366 \mathrm{cde}$ & 66.6 & 28.5 & 1.54 \\
\hline $\mathrm{Cl} 0972 \mathrm{bmr}$ & $6.015 \mathrm{def}$ & $10.135 \mathrm{ef}$ & 67.6 & 26.7 & 1.57 \\
\hline $\mathrm{Cl} 0919 \mathrm{bmr}$ & $5.717 \mathrm{ef}$ & $80649 \mathrm{f}$ & 64.8 & 30.2 & 1.48 \\
\hline $\mathrm{Cl} 0910 \mathrm{bmr}$ & $5.139 \mathrm{f}$ & $14.565 \mathrm{~b}$ & 66.5 & 28.0 & 1.50 \\
\hline
\end{tabular}

Letras distintas indican diferencias significativas $(p<=0.05)$.

Días a flor: Para medir la precocidad de los materiales se comparó los días que estos entran a floración. De las 16 variedades se encontraron diferencias altamente significativa $(p<0.0001)$.

Las variedades más precoces fueron CI 970, 914, 972, Centa S-3, 947 y 973, entre los 73 y 75 días $(P>0.05)$ no encontrando diferencias entre ellas. Las variedades más tardías fueron las $\mathrm{Cl} 910,925$ y 929 , con 85 y 81 días respectivamente $(p<0.05)$. Estos valores son reportados de los años 2010 y 2011 (Figura 2). 
Figura 2. Días de flor de 16 variedades de sorgo forrajero sembradas en parcelas experimentales en la época lluviosa en la Estación Experimental

La Lujosa en los años 2010 y 211.Letras distintas indican diferencias significativas $(p<=0.05)$.

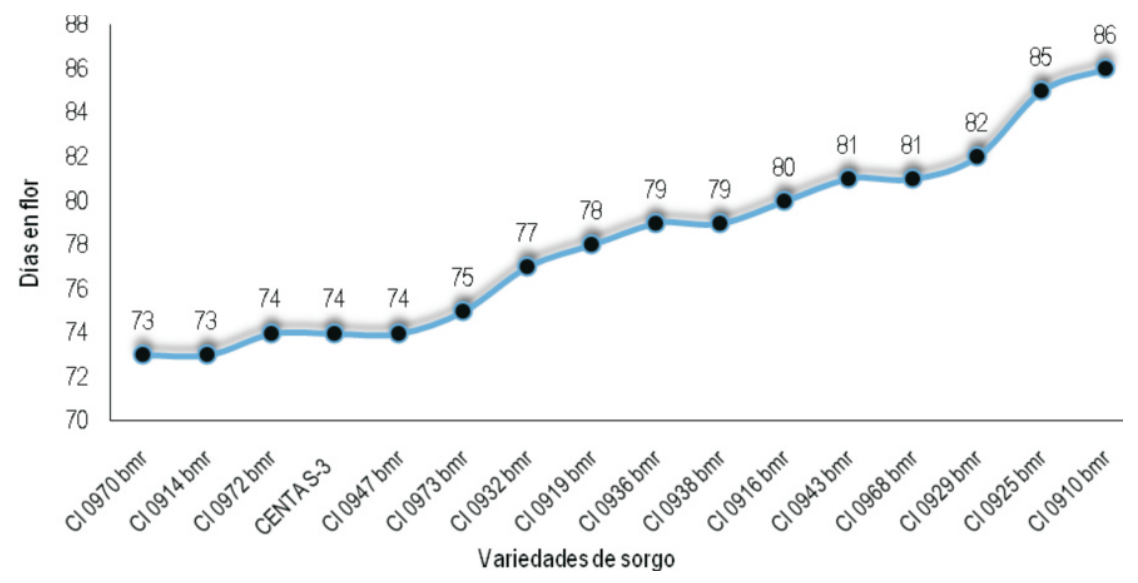

Altura de planta: Se encontraron las variedades CI 910 y Centa S-3 con la mayor altura de 262 y $251 \mathrm{~cm}$ respectivamente $(P<0.05)$, le sigue la variedad 932 con 240 $\mathrm{cm}$, y las variedades $938,916,925,973,929,968$ y 936, que estadísticamente presentan la misma altura (Figura 3 ).

Figura 3. Altura de planta de 16 variedades de sorgo forrajero evaluados en los años 2010 y 2011 en la temporada de invierno. Las variedades con mayor altura son las que pueden producir mayor contenido de forraje.

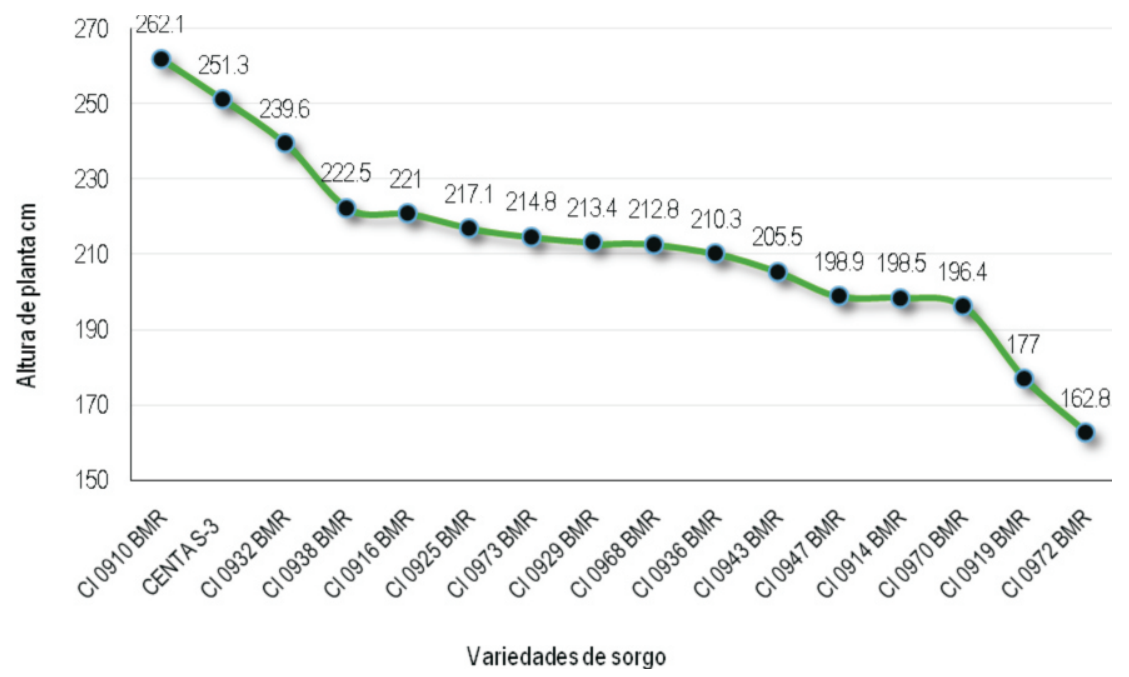


Producción de grano: en el período de agosto a diciembre de los años 2010 y 2011, se evaluó la producción de grano de 16 variedades de sorgo BMR, se encontró diferencias altamente significativas $(P<0.0001)$ en los rendimientos de producción de grano de estas variedades.

Al comparar la producción entre los dos años, se encontró que el año 2011, fue más productivo que el 2010 ( $P<0.05)$, debido a que hubo mayor distribución y presencia de lluvias durante ese año. En los rendimientos promedio de los dos años de la producción de grano de estas 16 variedades en kilogramos por hectárea, el mayor rendimiento de grano lo reporta el sorgo Centa S-3 con 3,549 Kg/ha, le sigue la variedad Cl 0947 con un rendimiento de 2,730 Kg/ha (Figura 4).

Las variedades $938,925,910,968,972,936,932,943$ y 970, presentaron rendimientos estadísticamente iguales $(P>0.05)$ con producciones entre 1,866 y $2,194 \mathrm{Kg} / \mathrm{ha}$ de grano. Las variedades que presentaron los rendimientos de grano más bajo fueron $973,919,914,916$ y 929 , que son entre 1,380 y $1,676 \mathrm{Kg} / \mathrm{ha}$.

Figura 4. Producción promedio de grano de 16 variedades de sorgo forrajero en la Estación Experimental La Lujosa de los años 2010 y 2011.
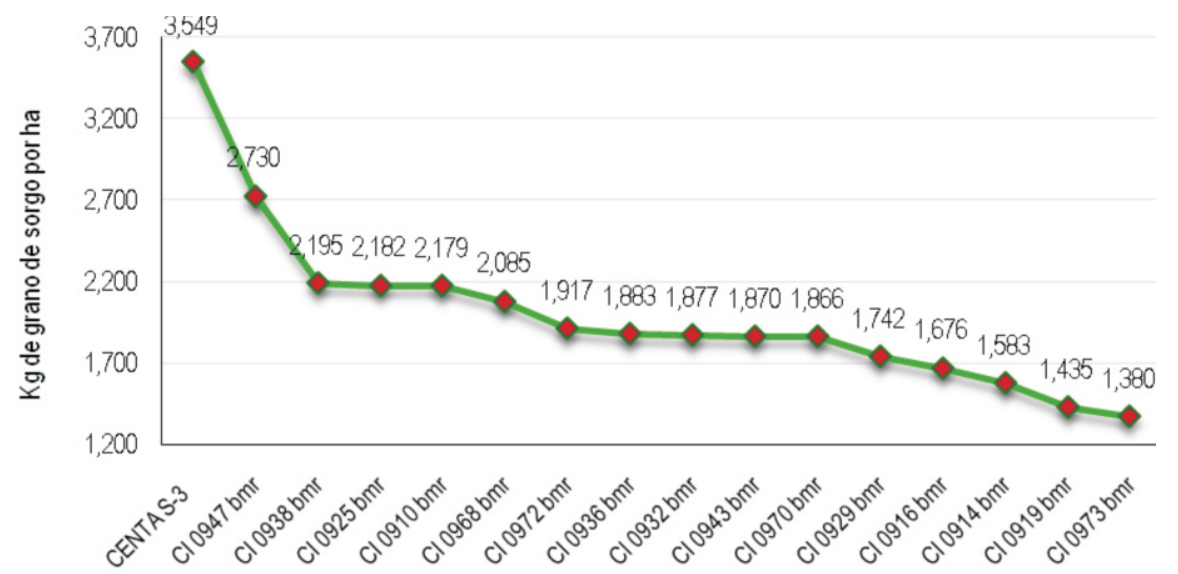

Variedades de sorgo

\section{DISCUSIONES}

De las cuatro parcelas experimentales, en los dos años de estudio, la variedad CENTA S-3 fue la que registró la mayor producción de forraje, seguido por la variedad $\mathrm{Cl} 0910$ bmr con 44,263 Kg de forraje por hectárea. Estadísticamente, las 
variedades $\mathrm{Cl}$ 0947, 943, 925, 932, 929, 938, 973, 916, 936, son iguales, no así las primeras dos variedades y la Cl0919, que reportó la menor producción de forraje con un valor de 30,357 Kg por hectárea. Las variedades con los menores valores de MSD fueron los sorgos 972, 919 y 910 . En el contenido de materia seca, la variedad Centa S-3 reportó el mayor valor de materia seca, le siguen las variedades 910, 947, y 925 . La variedad que presentó el valor más bajo en MS fue la 919 con 8,649 Kg/ha de MS.

El mayor valor reportado por las variedades en ENL fue la variedad de $\mathrm{Cl} 0925$ con $1.62 \mathrm{Mcal} / \mathrm{Kg}$ de MS, las variedades más baja fueron 919 y 947 con $1.48 \mathrm{Mcal} / \mathrm{Kg}$ de MS en ambos casos. La energía neta de lactancia es la energía contenida en la leche producida por el rumiante. La calidad del forraje es medida por el porcentaje de NDT y la ENL, a mayor valor de estas referencias nutricionales, el forraje es de mejor calidad para el animal.

El sorgo que presentó el mayor porcentaje de NDT fue la variedad $\mathrm{Cl} 0925$ con $68.8 \%$, y le siguen las variedades $972,914,910,970$ y 973 . El material con el porcentaje más bajo de NDT fue la variedad $\mathrm{Cl} 943$ con $62 \%$. Los valores más bajos de NDT, MS (\%) y ENL de los materiales de sorgo sometidos al estudio, fueron mayores que los reportados del comité internacional de nutrición animal con $23 \%$ de MS, $63 \%$ de NDT y $1.42 \mathrm{Mcal} / \mathrm{Kg}$ de ENL respectivamente. La variedad que produjo la mayor cantidad de grano fue el Centa S-3, a pesar de las discrepancia en las lluvias en los años 2010 y 2011, su producción fue mayor de los $3,500 \mathrm{~kg}$ de grano de sorgo por hectárea.

\section{CONCLUSIONES}

1. Los mejores materiales de sorgo encontrados en las parcelas experimentales fueron las variedades Centa S-3, Cl0910 y Cl0925, ya que mostraron las mejores características de desempeño como producción de biomasa (materia fresca), producción de materia seca digerible, nutriente digestible totales y energía neta en la lactancia.

2. La variedad que obtuvo la mayor producción de grano fue Centa S-3, seguida de Cl0947, Cl0938, Cl0925 y Cl0910, produciendo más de 2,000 Kg de grano por hectárea.

3. Las variedades que registraron la mayor altura de planta fueron $\mathrm{Cl} 0910$ y Centa S-3, en cuanto precocidad o días a flor, las variedades más precoces fueron Cl0970, Cl0914, Cl0972 y Centa S-3.

4. Las mejores variedades fueron Centa S-3, Cl0910 y CI0925, por lo que deben liberarse para estar al alcance de los pequeños agricultores. 


\section{AGRADECIMIENTOS}

Se agradece a la Dirección de Ciencia y Tecnología Agropecuaria de la Secretaría de Agricultura y Ganadería, por el apoyo técnico y económico para la conducción y elaboración del estudio. También se reconoce el agradecimiento a la Estación Experimental La Lujosa, y al equipo que apoyo con la conducción del experimento durante los dos años de estudio.

\section{BIBLIOGRAFÍA}

Browning, C., \& Lusk, J. (1966). Comparison of feeding value of corn and grain sorghum silages on the basis of milk production and digestibility. Dairy Science Department, Mississipi State University, State College.: Journal of Dairy Science, 49(12): 1511-1514.

Díaz, L. A. (2011). Evaluación comparativa de sorgo nervadura café (Pampa Centurión) y sorgo nervadura blanca (Sureño) para la producción de ensilaje, Zamorano, Honduras. Tegucigalpa, Honduras: Zamorano.

IICA. (2010). Experiencias con el bono tecnológico productivo en las comunidades de Jacaleapa y San Juan de Linaca, El Paraíso, Honduras. Tegucigalpa, Honduras: Instituto Interamericano de Cooperación para la Agricultura.

Rodriguez, L. (18 de Junio de 2012). Cultivo de sorgo crece en Honduras. Recuperado el 30 de octubre de 2012, de El Heraldo:

http://www.elheraldo.hn/Secciones-Principales/Economia/Cultivo-desorgo-crece-en-Honduras

SAG. (marzo de 2010). Cadena de granos básico. Recuperado el 2012 de octubre de 23, de Secretaría de Agricultura y Ganadería:

http://www.sag.gob.hn/files/Agronegocios/Oportunidades/granos_ basicos.pdf

SAG. (2010). Estaciones Experimentales. Recuperado el 01 de noviembre de 2012, de Dirección en Ciencia y Tecnología Agropecuaria:

http://www.dicta.hn/programa-de-estaciones-experimentales-.html

Sandoval, F. Y. (2011). Análisis de mercado de semilla de maíz mejorada en Honduras. Honduras: Zamorano.

Urquiza, A. C. (2001). El sorgo forrajero en producción animal. Recuperado el 03 de noviembre de 2012, de Producción animal: www.produccion-animal.com.ar. Valencia, R. C. (2011). Evaluación preliminar de híbridos de sorgo (Soghum Sp) para forraje 2012. San Salvador, El Salvador: INTSORMIL. 